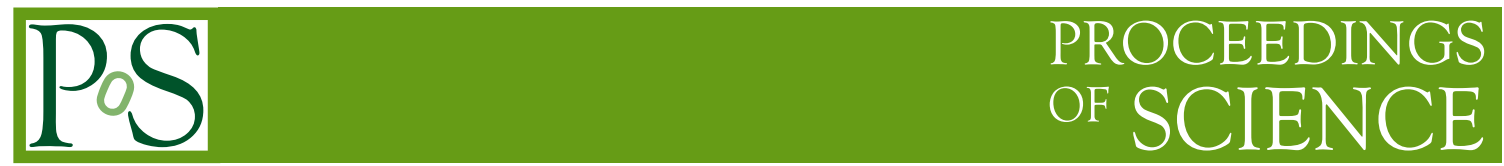

\title{
Primordial non-Gaussianity, present status and future prospects
}

\section{Sabino Matarrese*}

Dipartimento di Fisica e Astronomia G. Galilei, Università di Padova

E-mail: sabino.matarreseapd.infn.it

I will review both the general problem of the search for non-Gaussian signatures in cosmological perturbations, originated from inflation in the early Universe. I will discuss this issue both from the theoretical point of view and in connection with constraints coming from recent observations and future prospects for observing/constraining them.

Frontiers of Fundamental Physics 14 - FFP14,

15-18 July 2014

Aix Marseille University (AMU) Saint-Charles Campus, Marseille

${ }^{*}$ Speaker. 